Cambridge University Press

978-1-107-01197-7 - Understanding Sponsored Search: Core Elements of Keyword Advertising Jim Jansen

Excerpt

More information

\title{
A Context for Sponsored Search
}

\author{
Context is the surroundings, circumstances, environment, background, \\ or settings which determine, specify, or clarify the meaning of an event.
}

Wiktionary, 2010 [1]

You must always consider the context within when a given phenomenon occurs to derive any meaning from it. As the Wiktionary quote states, the context is what clarifies the meaning of an event [1].

\section{Let's Place Ourselves in the Proper Context}

You are the owner of a small- to medium-size business that sells some product or service. Pick any product or service that interests you. We will use a framing shop as an example throughout this book. Our framing shop is called Faster Frames.

You have a brick-and-mortar storefront, but you decide that you also want to sell your product (or service) online to better serve the potential customers in your immediate area and to potentially market to an audience beyond your specific geographical area. What do you do?

Well, first you need some sort of virtual storefront. Traditionally, this has been a Web site, either built in-house or contracted out to a company that provides this service. However, it can also be a virtual presence on a number of social media platforms where some aspect of business occurs. For our framing shop, we have created a nice Web site showing our frames and our framing service.

What now?

It is rare for a brick-and-mortar store to have large numbers of potential customers just walk in. Most often, one has to do some marketing to alert the potential consumer base that the business exists. Certainly, if your product, pricing, performance, place, and people provide value to consumers, marketing is much easier, as your business will probably have a good brand image with the potential customer base. However, there are typically competing businesses that offer similar (or identical) products in one's geographical area. So, even the top companies typically have to engage in some sort of marketing and advertising efforts. This is certainly true for our framing shop, as there are several national competitors, as well as numerous local franchises and several mom-and-pop stores. 
Cambridge University Press

978-1-107-01197-7 - Understanding Sponsored Search: Core Elements of Keyword Advertising Jim Jansen

Excerpt

More information

The situation is even more competitive in the virtual world. There could potentially be dozens, hundreds, even thousands of companies competing in the same market. You may even find that many of these companies have been competing with your brick-and-mortar store in your own geographical area.

So whether you want to or not, you are competing in the online world!

Given that framing is a service as well as a product, as long as we have a shipping and receiving service, there is no reason we cannot compete nationally for the high-end market.

Each of these competing businesses has some sort of online presence, a virtual storefront. It is rare for a Web site to have large numbers of potential customers just randomly arrive at their site. Just as in the brick-and-mortar world, businesses must do some marketing to get new customers to their online storefronts. Certainly, given the competitive marketplace, this is true for our framing shop.

Marketing on the Web hinges on a few key technology services. Social media sites are important as commercial marketing outlets and are especially important as viral marketing. However, the big marketing guns in the online world are the search engines. Of course, the social media sites and the search engines may be the same entities.

\section{How do these Search Engines Impact the Business?}

For years, search engines have been the major gateways to the Web. With their legions of software agents indexing Web pages, acres of computers for storing terms and metadata from these Web pages, and intuitive interfaces for searching, these search engines are the workhorses of the Web. They are critical navigational elements that enable people in their role as potential consumers (along with many other roles) to leverage the vast content on the Web. In other words, the major Web search engines are value enhancers. The information on these Web sites would be of little value if few people could find them.

Potpourri: Although it is now obvious that search engines and the techniques they employ are critical tools for using the Web, it was not always so clear-cut.

The original manuscript discussing the highly important Google ranking algorithm was rejected by the Special Interest Group on Information Retrieval (SIGIR) Conference, which is the leading information retrieval conference in academia.

In defense of the SIGIR reviewers, the paper was not clearly written, which is an example of the important relationship between function and form. One cannot neglect either.

A version of the paper was eventually published in an academic journal and has subsequently become one of the most highly cited papers in the informationretrieval field (see [2]).

Why has the Web had such a big impact?

One reason may be the theory of affordances [3], which posits that people view the world in terms of both object shapes (i.e., including spatial relationships) and object 
Cambridge University Press

978-1-107-01197-7 - Understanding Sponsored Search: Core Elements of Keyword Advertising Jim Jansen

Excerpt

More information

possibilities for action (i.e., affordances). This perception of object possibilities helps drive people's action (i.e., perception drives action). Sometimes these affordances are what designers intended. At other times, people bring their own affordances to a technology, which has certainly occurred with the use of the Web.

So for our virtual business, we have to get our Web site indexed by the major search engines. Luckily, this is fairly easy to do. Sometimes, we have to do nothing. Other times, we may have to request the search engine to index our site. Given that we are somewhat technically suave, we quickly get the Web site for our framing shop indexed by major search engines.

Once indexed, though, our real challenge begins. The screen space on any computer or computing device is limited. There is only so much space (or screen real estate) to show information. So, when a searcher submits a query to a search engine, there are only so many results that a search engine can display. Let's say this number of results is ten.

Actually, ten is an exaggeration, as there are typically fewer than ten results that appear above the fold (the portion of the screen that first appears to the user where the user does not have to scroll down). Most users do not scroll down [4].

So, a potential customer submits a query that relates directly to your business. Although you might imagine that one of the results would be for your site, there is no guarantee. It is rare for a business to have a market alone to itself. Rather there may be from dozens to hundreds to thousands of businesses (all with viable products for the potential customer) competing for one of those ten result slots.

Your business wants to be one of these ten slots on the first page of results, which is where most of the consumer traffic is; actually, you want to be at the top of this list. With each business equally viable, the search engine uses a variety of factors to rank which businesses get one of those ten slots. However, you have only a limited idea of what factors the search engine uses for ranking, so it is somewhat of a crapshoot.

What other options does your business have to get on this page of results? One answer lies with sponsored search (a.k.a. keyword advertising, paid search, payper-click). Sponsored search is the process in which advertisers pay to have their advertisements appear on a search engine results page in response to a query from a searcher. In sponsored search, advertisers pay search engines for traffic from the search engine to their Web sites. With sponsored search, major Web search engines have significantly altered online commerce.

Potpourri: Sponsored search can come in various forms; the most common is pay-per-click.

The Search Engine Marketing Professional Organization (SEMPO) defines pay-per-click as a model of online advertising in which advertisers pay only for each click on their ads that directs searchers to a specified landing page on the advertiser's Web site.

Let's say that our framing shop does not show up in the top ten for the query "frame shops" in our area. Then, we are really going to depend on sponsored search as an advertising medium. 
Cambridge University Press

978-1-107-01197-7 - Understanding Sponsored Search: Core Elements of Keyword Advertising Jim Jansen

Excerpt

More information

\section{How Do We Leverage Sponsored Search to Market Our Business?}

The major search engines have sponsored search platforms where one can establish an account within a short amount of time. There are a couple of implementations of sponsored search. We are going to assume that we signed up for the version where we pay the search engine when someone clicks on one of our ads (referred to as payper-click). We might sign up for accounts on multiple search engines. We sign up for a sponsored search account on a major search engine for our framing shop.

Our goal in this sponsored search effort is the same as in all advertising endeavors. We aim to acquire new customers at a cost that permits us to make a profit on the products and services that we sell to these customers.

\section{Once Done with this Administration, What Do We Do?}

The first step is to select some words that we believe potential customers will use when they would want to find our business on the Web. Using the search engine's sponsored search platform, we then add these keywords to our account. Typical keyphrases for our framing shop could be "frames," "framing," and "custom framing."

We decide what advertisements we want to appear when a searcher enters one of these keywords. Using the search engine's sponsored search technology, we create the advertisements on our account, linking ads to the keywords that we selected.

Let's assume that we create the following ad for our framing shop:

\section{Faster Frame}

\$50 Off Custom Framing Coupon

FASTER FRAME Satisfaction Guaranteed

www.fasterframe.com

Once we have a sponsored search account established and running, our ads can appear on Web pages, cell phones, tablets, and many other devices. These ads can take potential customers to our Web site or provide them a means to click the ad and call our business or visit some other site related to our business, such as one of our social media sites.

\section{Okay, How does the Search Engine Make Money?}

The search engine company does not provide this marketing service for free. We offer to pay the search engine a set amount for each keyphrase, which is called a bid. It is called a bid because there may be (and probably are) several other businesses wanting to show their ads for the same keywords that we want to show our ads. Each business offers a bid, and the laws of supply and demand take over. The more businesses that want to bid on a certain keyword, the higher the bid price to get your ads to show on the search engine results page (SERP), with the critical resource being screen real estate.

The bid price is the maximum amount that we will pay the search engine when someone clicks on our ad. Generally, what we actually pay is the same as the bid price. Sometimes lower. Never higher. 
Cambridge University Press

978-1-107-01197-7 - Understanding Sponsored Search: Core Elements of Keyword Advertising Jim Jansen

Excerpt

More information

This bidding process thus involves some decisions on the part of each business namely, what cost the business is willing to shoulder for its advertisements. This is rather difficult to determine at first, but once we get some historical data, we can finetune our bids and budget. For our framing shop, we will set a maximum bid price of $\$ 1.00$ per click.

So, we have our account set up, our keywords entered, our ads crafted, and our bids set. We are ready to roll with our sponsored search effort! We activate our account, and typically within minutes, our ads are set to appear.

It is really that simple. One could be running a sponsored search campaign in a matter of minutes. However, there are a lot of complex questions, assumptions, and issues underlying this seemingly simple setup.

This book addresses those complexities.

Potpourri: Sponsored search is a poster child for the concept of technological innovation, which consists of three stages, in a self-reinforcing cycle.

The three stages are: (1) a creative and feasible idea, (2) a practical implementation and application, and (3) the diffusion of this technology throughout society [5, p, 27].

It is really quite amazing that in the period of less than one decade, sponsored search grew from conceptualization to a multibillion-dollar enterprise, directly affecting millions of businesses and billions of people. It has shaped the Web as we know it.

In addition to its direct impact, it probably has had second-order effects of several billion dollars more [6].

\section{What are these Complex Questions, Assumptions, and Issues?}

Let's start with the seemingly simple step of selecting keywords. Which keywords do we select? How many do we select? Why would we select these? The selection of keywords is a critical step in the sponsored search process, and there are literally thousands of guides, checklists, and tools to aid us. However, there are limited documents that address the underlying theories and models to inform us what these guides, checklists, and tools are based on, or why certain keywords work and others do not. In this book, we examine these underlying theoretical aspects.

It is a similar case with advertisements. Advertisements in sponsored search systems are typically short, with just a few lines of text (with maybe other elements, such as a small image). Like with keywords, there are numerous checklists and suggestive guides to craft sponsored search advertisements. But why do these suggestions work? Why do certain ads get the potential customer's attention whereas others do not? What causes potential customers to click on certain ads but not click on others that appear for the same keyword?

What about the bidding? It would seem, at first glance, to be a simple process of deciding how much the business would like to spend. In actuality, our bids are part of a complex and intricate online auction process, with multiple competing interests (i.e., ours, our competitor's, and the search engine's). 
Cambridge University Press

978-1-107-01197-7 - Understanding Sponsored Search: Core Elements of Keyword Advertising Jim Jansen

Excerpt

More information

6

Understanding Sponsored Search

These are some of the issues with major components of sponsored search.

There are other related components to consider:

- Customer Component: How can we determine what the customer is really after? How will the customer behave when shopping online? What causes the customer to make a purchase?

- Marketing Component: How does our business engage in the sponsored search process? What are the business's overall goals and objectives?

- Advertising Component: How can we leverage sponsored search to achieve the objectives of our advertising efforts?

- Branding Component: What is the image that we want for our business in the online marketplace? Can we leverage sponsored search to increase our brand worth?

\section{Conclusion}

It is for the understanding of these issues that this book is written. No tactics, no checklist, and no implementation advice will address these questions. For this, we must venture into the place of theory and models.

Let's begin.

\section{References}

[1] Wiktionary. 2010. Context. Retrieved January 15, 2011, from http://en.wiktionary.org/wiki/ context.

[2] Brin, S. and L. Page . 1998. "The Anatomy of a Large-Scale Hypertextual Web Search Engine." Computer Networks and ISDN Systems, vol. 30(1), pp. 107-117.

[3] Gibson, J. J. 1977. "The Theory of Affordances." In Perceiving, Acting and Knowing, R. Shaw and J. Bransford, Eds. Hillsdale, NJ: Erlbaum.

[4] Jansen, B. J. and Spink, A. 2003. "An Analysis of Web Information Seeking and Use: Documents Retrieved Versus Documents Viewed.” In 4th International Conference on Internet Computing, Las Vegas, NV, pp. 65-69.

[5] Toffler, A. 1970. Future Shock. New York: Random House.

[6] Johnson, C. H. 2009. Google's Economic Impact United States 2009. Mountain View, CA: Google. 
Cambridge University Press 978-1-107-01197-7 - Understanding Sponsored Search: Core Elements of Keyword Advertising Jim Jansen

Excerpt

More information 
Cambridge University Press

978-1-107-01197-7 - Understanding Sponsored Search: Core Elements of Keyword Advertising Jim Jansen

Excerpt

More information

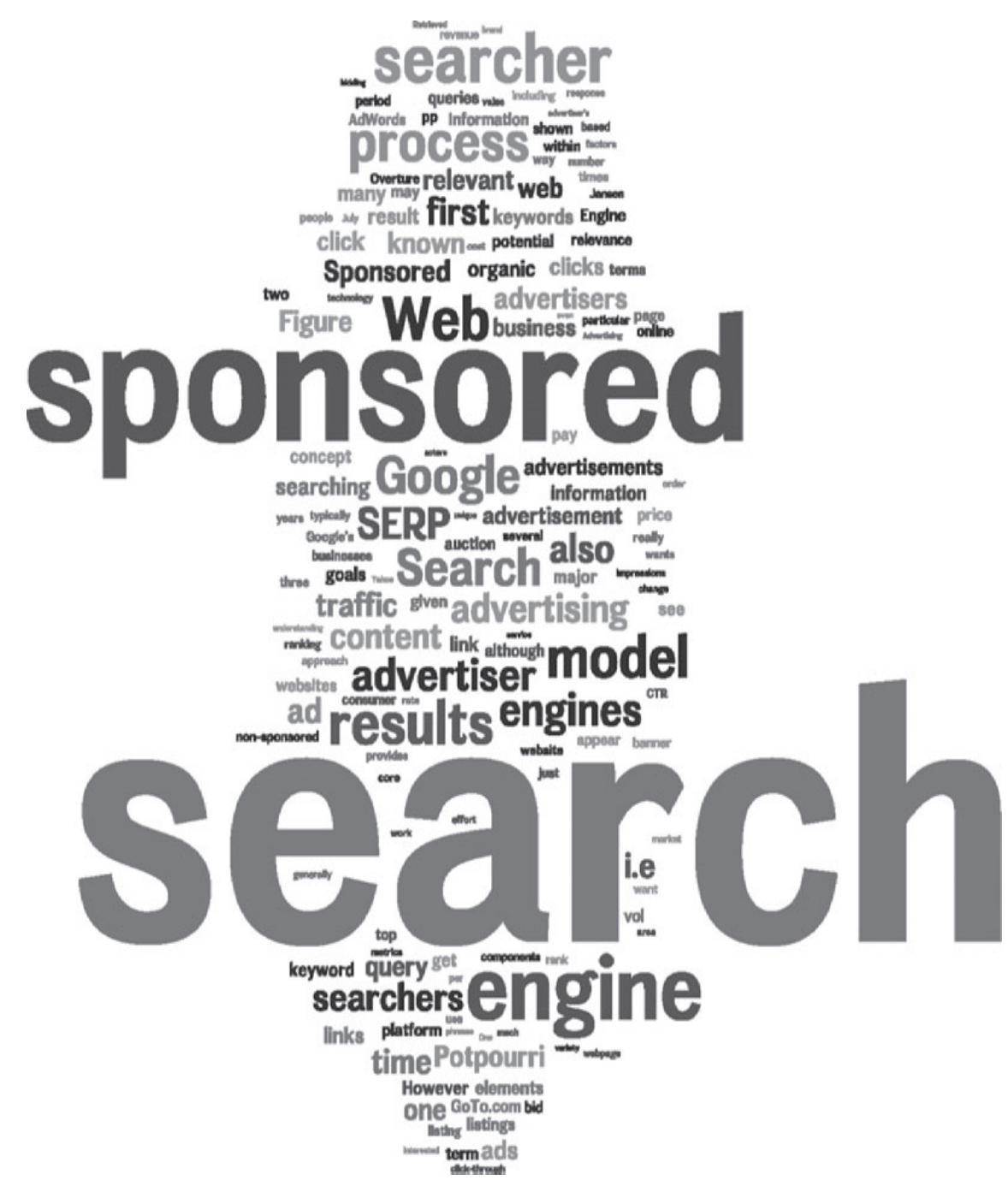

Word cloud generated by Wordle 
Cambridge University Press

978-1-107-01197-7 - Understanding Sponsored Search: Core Elements of Keyword Advertising Jim Jansen

Excerpt

More information

\title{
Modeling the Process of Sponsored Search
}

\author{
This is an opening shot of changing the search engines \\ from white pages to yellow pages. \\ Bill Gross,
}

1998 as quoted in: Sullivan, D., The Search Engine Report [1].

In our framing shop example of setting up our sponsored-search account, we touched on a process by example without a real appreciation for the underlying complexity of the technology and human interaction inherent in sponsored search. What is the technology that makes the whole process work? What is the business model of sponsored search? Who are the major actors in sponsored search? What are the goals and motivations of these actors? For answers to these and other questions, we examine the sponsored-search model.

It is important to develop a generic model of the sponsored-search process. A model is a representation of a complex natural process, simplifying but still embodying the essential attributes. In other words, a model allows us to peel away the unnecessary to get at the critical elements.

Adding some flavor to our model, it is worthwhile to begin with a brief introduction of the beginnings of sponsored search, which will shed light on how we got to where we are. Bill Gross [1] was right that the introduction of advertising in the search engine results page was to change everything. Finally, we end this chapter with some of the conceptual jargon of sponsored search.

\section{Historical Review of the Early Years of Sponsored Search}

The first attempt at something like sponsored search was by the search engine OpenText in 1996 [2]. However, the experiment did not go well for a variety of reasons, perhaps the main one being the desire of searchers to not commercialize the searching process. In fact, the company got so many complaints that OpenText removed the feature within a matter of weeks. 
Cambridge University Press

978-1-107-01197-7 - Understanding Sponsored Search: Core Elements of Keyword Advertising Jim Jansen

Excerpt

More information

Potpourri: To illustrate how early the concept of sponsored search was introduced, the very first occurrence of Web advertising that I could document occurred on October 27, 1994, when Hotwired signed fourteen banner advertisements to display on their site $[3$, p. 6]. On that day, the Web became a real commercial medium [3].

Supposedly, AT\&T was the first company to have their banner ad displayed, although other companies also can lay claim to the fame, including Club Med, MCI, Volvo, and Coor's Zima.

Disclaimer: Other Web-based magazines (a.k.a., Webzines) lay claim to being the first to market with banners ads, including Global Network Navigator, Virtual Journal, Synapse, Medio, and Chaos Control. All, however, were in the 1993 or 1994 timeframe.

Regardless, Rick Boyce, Hotwired's director of business in 1994, is credited with pioneering the idea of banner ads as a major business concept for online companies.

Here (supposedly) is something close to what that first banner ad looked like (Figure 2.1).

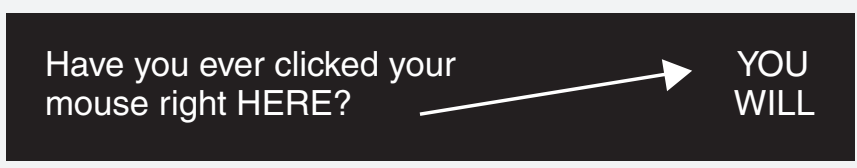

Figure 2.1. Image of first banner advertisement.

It reminds me of one of the cheesy roadside billboards that one sees along interstates in the United States promoting those tourist traps just beyond some state line.

On February 21, 1998, GoTo.com launched a sponsored-search model in which the search engines ranked "Web sites based on how much the sites are willing to pay to be placed at the top of the search under a real-time competitive bidding process [2]." Advertisements for these Web sites appeared on the search engine results page, and the ad displayed based on the searcher was actively seeking at the time.

The conceptualization was relatively straightforward, with a transparent ranking factor (i.e., money), advertisers bidding on exact phrases, and editors checking for relevance. This concept is a first-price auction, where the top bidder gets the top advertising position. GoTo.com also provided nonsponsored listings, provided by Inktomi.com.

Potpourri: GoTo.com was the rebranded search engine, World-Wide Web Worm, which was the first Web search engine.

Created in September 1993 by Oliver McBryan at the University of Colorado, the World-Wide Web Worm is the grandfather of all Web search engines. It started it all! 